\title{
Hybrid Biomedical Intelligent Systems
}

\author{
Maysam Abbod, ${ }^{1}$ Jiann-Shing Shieh, ${ }^{2}$ and Hak-Keung Lam $^{3}$ \\ ${ }^{1}$ School of Engineering and Design, Brunel University, Uxbridge UB8 3PH, UK \\ ${ }^{2}$ Department of Mechanical Engineering, Yuan Ze University, Chung-Li 32003, Taiwan \\ ${ }^{3}$ Division of Engineering, King's College London, London WC2R 2LS, UK \\ Correspondence should be addressed to Maysam Abbod, maysam.abbod@brunel.ac.uk
}

Received 20 March 2012; Accepted 20 March 2012

Copyright ( $) 2012$ Maysam Abbod et al. This is an open access article distributed under the Creative Commons Attribution License, which permits unrestricted use, distribution, and reproduction in any medium, provided the original work is properly cited.

Computerised systems are one of the fastest growing areas within medicine at present, both in the clinical setting in hospitals and in research and development (R\&D). The importance of such systems for patient management is growing steadily and plays an increasingly important role in treatment and diagnosis. Intelligent systems are becoming the core for such system where they provide more rapid and precise patients' management, aid diagnosis, and improved outcome. Furthermore, intelligent systems benefit the health care system through improved accuracy and more costeffective solutions.

Initially, singular approaches were used, but increasingly this has moved towards hybridisation, in particular that of artificial intelligence techniques. The current emphasis is on hydride models (quantitative/qualitative) and hybrid intelligent algorithms (fuzzy logic, neural networks, and neurofuzzy systems) applied into biomedical applications. The purpose of this special issue is to promote research and developments of the best work in the field of hybrid intelligent systems for biomedical applications.

One paper in this special issue addresses an improved intuitionistic fuzzy cross-entropy for medical pattern recognition. The improved nonprobabilistic entropy approach supports doctors examining the work of the preliminary diagnosing. Another paper is concerned with classifying high-dimensional patterns using fuzzy logic discriminate network. The technique is based on an adaptive network of fuzzy logic connectives to combine class boundaries generated by sets of discriminate functions. Another different paper investigates the use of an intelligent dynamic MRI
(DMRI) system for automatic nasal tumour detection. The dynamic MRI is one of the major tools for diagnosing nasal tumours in recent years. The purposed system detects and enhances the tumour region in DMRI automatically by using Adaboost, SVM, and Bayes-Gaussian classifier.

One other paper presents a novel technique for identifying patients with ICU needs using hemodynamic features. The system can classify the types of patients and their ICU need based on hemodynamic features such as heart rate and blood pressure. Further analyses are done based on the initial measurements such as circle criterion, estimation error criterion, Poincare plot deviation, and autonomic response delay criterion. All the features are utilised to classify the patient using a fuzzy system. There is a paper that investigates the effect of the initial random weight of the neural network on the accuracy of the trained model. Hip fracture data were used to generate a model to predict the patient status using a neural network model. The initial weights of the neural network have been optimised using genetic algorithm. Results show that the initialisation process has a big effect on the accuracy of the trained model. Another paper addresses the use of neural networks for PET volume analysis and segmentation; different images were used to train neural network for classifying tumour data on PET images. The developed classifier shows that the system has a high accuracy such that a single tumour pixel can be detected and classified accurately.

Maysam Abbod Jiann-Shing Shieh Hak-Keung Lam 

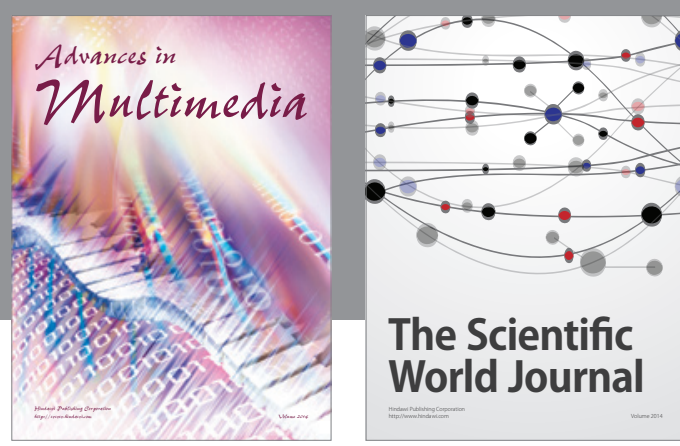

The Scientific World Journal
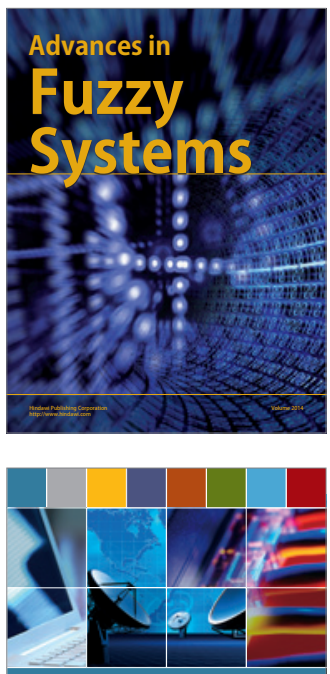

Computer Networks and Communications
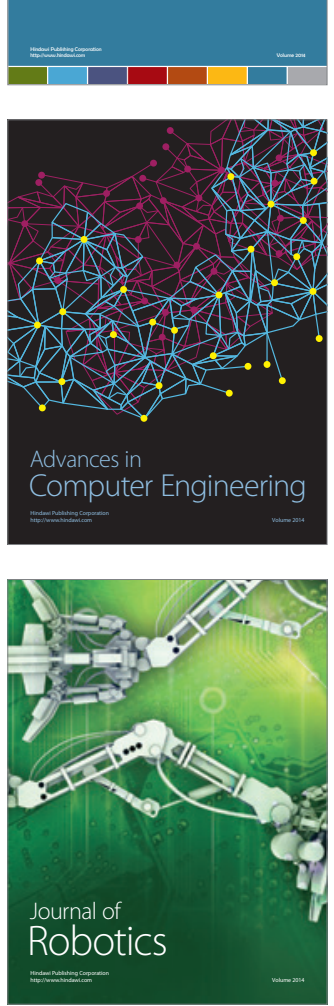
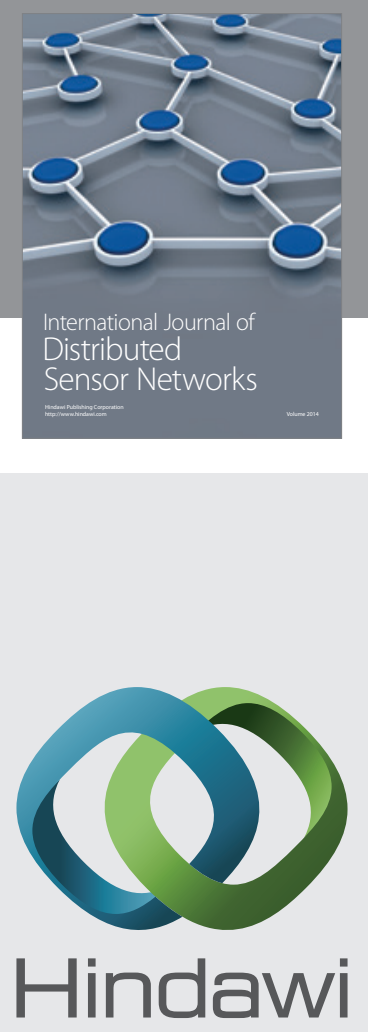

Submit your manuscripts at

http://www.hindawi.com
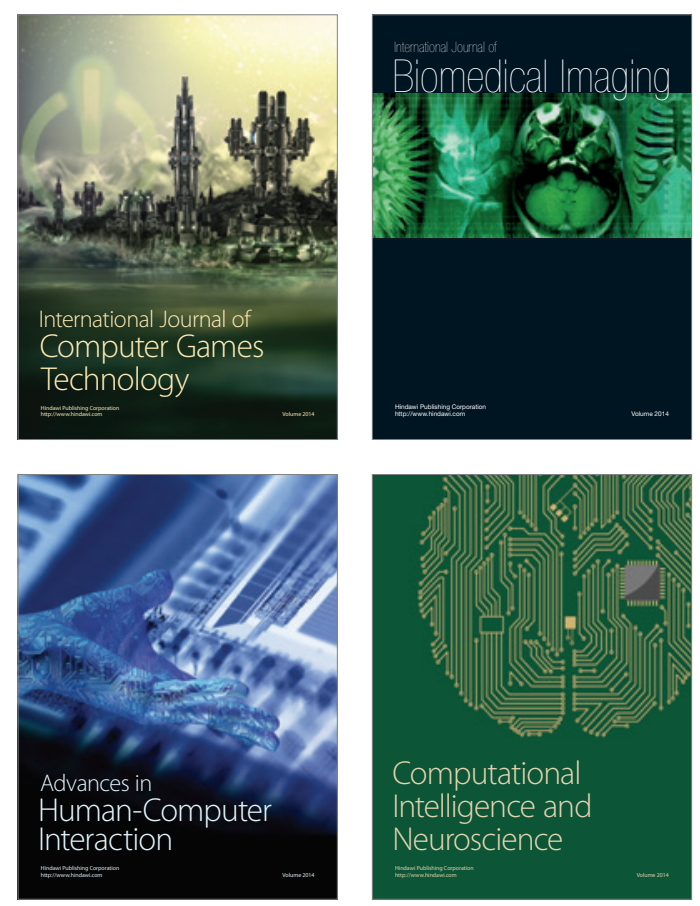
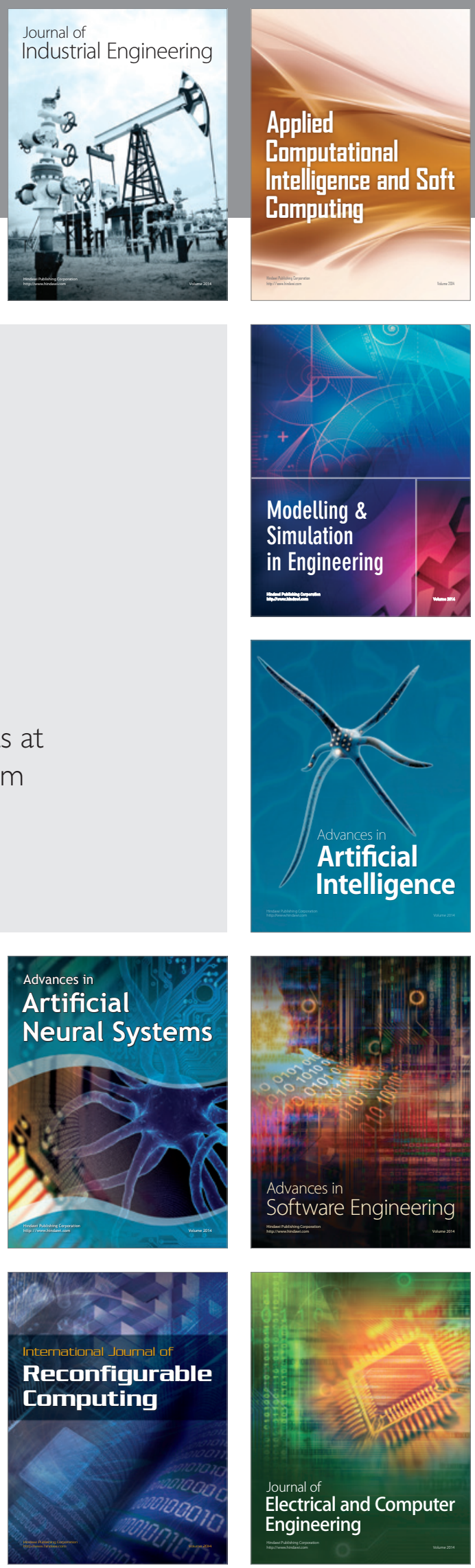